Chirurg 2012 $\cdot 83: 307-308$

DOI 10.1007/s00104-011-2187-4

Online publiziert: 15. März 2012

๑) Springer-Verlag 2012

\author{
J. Fritzmann · A. Ulrich · M.W. Büchler \\ Chirurgische Universitätsklinik, Klinikum der Universität Heidelberg
}

\title{
Chirurgie in Partnerschaft
}

Liebe Kolleginnen und Kollegen,

es ist bereits Tradition, dass die Ausgabe von Der Chirurg im Monat des Jahreskongresses der Deutschen Gesellschaft für Chirurgie von deren Präsidenten konzipiert wird. So lehnt sich dieses Heft an das Motto und die Thementage des diesjährigen Kongresses an. Das Motto lautet: Chirurgie in Partnerschaft, was auch gleichzeitig den Thementag am Mittwoch des Kongresses darstellt. Die Chirurgie, getragen von der Deutschen Gesellschaft für Chirurgie und ihren zehn großen Fachgesellschaften, ist heute mehr denn je eine zentrale Disziplin der Medizin. Kollegiale Partnerschaft mit den uns zuweisenden und zugeordneten ärztlichen Kollegen anderer Disziplinen macht die moderne Chirurgie zu einer von uns und unseren Patienten dringend erwünschten interdisziplinären Kraft. Eine besonders enge Zusammenarbeit verbindet uns Chirurgen naturgemäß durch unsere tägliche Arbeit im Operationssaal mit den Kollegen der Anästhesie. Eine gute Zusammenarbeit führt hier zu einem deutlich besseren Outcome für die gemeinsamen Patienten. Ein "Alleingang" wäre in der heutigen modernen Medizin undenkbar. Interdisziplinarität führt aber auch zu Unklarheiten in der Kompetenzverteilung und zu unerwünschten „Umarmungen“. Hier sei nur exemplarisch auf die Behandlung von Klappenfehlern durch Kardiologen und Kardiochirurgen hingewiesen.

\section{》) Ein "Alleingang" ist in der modernen Medizin undenkbar}

Als ein unverzichtbarer Partner im klinischen Alltag hat sich die Pflege herauskristallisiert. Durch neue Organisations- formen, entwickelt von beiden Berufsgruppen gemeinsam und auf Augenhöhe, ist eine fortschrittliche und effiziente Krankenhausstruktur mit zentralem Patienten-, Aufnahme- und Entlassmanagement erst möglich geworden.

Chirurgen sind nicht nur Partner ärztlicher und nichtärztlicher Kollegen in der Klinik, sondern auch Partner der Patienten, Partner und Teil der Wissenschaft und Forschung, aber auch Partner der Industrie.

Als weitere Schwerpunkte und $\mathrm{Na}$ mensgeber für die Thementage des Kongresses und dieses „Kongressheftes“ wurden „Forschung und Studien“, „perioperative und Intensivmedizin“ sowie „Organisation und Management" gewählt.

Die Entwicklung unseres Faches im Bereich Forschung und Studien ist überaus dynamisch. In der Chirurgie wird überdurchschnittlich viel und erfolgreich geforscht. Das breite Spektrum der Chirurgie ermöglicht Forschung in den verschiedensten Bereichen wie beispielsweise der Transplantationschirurgie mit der Entwicklung neuer Materialien zum Ersatz von Organen oder Organbestandteilen oder in der minimal-invasiven Chirurgie mit immer weiterführenden technischen Entwicklungen. Dies alles führt zu einer stetigen Verbesserung in der Behandlung der uns anvertrauten Patienten. Auch die Grundlagenforschung und die Initiierung und Durchführung klinischer Studien ist ein fester Bestandteil der heutigen akademischen Chirurgie. Gerade die Etablierung des Studienzentrums der Deutschen Gesellschaft für Chirurgie hat durch nachhaltige Unterstützung klinischer Forschung in der Chirurgie zu einem deutlichen Anstieg der Qualität klinischer chirurgischer Studien aus Deutschland und zur internationalen Konkurrenzfähigkeit beigetragen. Dem 
immer wieder beschworenen Nachwuchsmangel in der Chirurgie kann durch frühe Einbindung zukünftiger und junger Kollegen in die chirurgische Forschung entgegengewirkt werden und zu einer erheblichen Attraktivitätssteigerung unseres Faches beitragen.

Die perioperative Medizin umfasst verschiedenste Bereiche in der Behandlung unserer Patienten, wie die prästationäre Patientenbetreuung, Aufklärung und Einwilligung, Fragen der Patientensicherheit, Hygiene, Antibiotikaprophylaxe, Schmerztherapie und die postoperative intensivmedizinische Behandlung. Der Intensivmedizin gebührt dabei eine herausgehobene Stellung, da deren Weiterentwicklung eine Voraussetzung für die chirurgische Therapie immer komplexerer und aufwendigerer Operationsverfahren ist. Die fortschreitende Entwicklung unseres Faches Chirurgie ist damit eng an die Entwicklung der Intensivmedizin gekoppelt. Daher sollte diese auch in $\mathrm{Zu}$ kunft von Chirurgen mitgestaltet werden.

Zudem müssen wir unsere Anstrengungen in Hinblick auf eine evidenzbasierte Medizin auch weiter konsequent auf die perioperative Medizin - exemplarisch sei hier die „Fast-Track-Rehabilitation“ genannt - ausweiten. Geringere Komplikationsraten, eine kürzere Krankenhausverweildauer und eine größere Patientenzufriedenheit werden der Lohn für diese Anstrengungen sein.

Hochaktuelle Themen sind weiterhin Organisation und Management chirurgischer Abteilungen unter den Bedingungen des Kostendrucks im Gesundheitswesen. Durch die Ökonomisierung der Krankenhäuser werden immer häufiger Zielvereinbarungen zwischen chirurgischen Abteilungsleitern und der Verwaltung geschlossen. Über die Reduzierung der privatärztlichen Liquidationsrechte und Einführung von Boni bei Erreichen der Zielmarken wird die Beziehung zwischen Arzt und Patient auf eine harte Probe gestellt, da medizinische Entscheidungen auch häufig aus einem ökonomischen Blickwinkel hinterfragt werden müssen. Um den Verwaltungen in Zukunft auf Augenhöhe entgegentreten zu können, sollten sich chirurgische Führungskräfte auf dem Gebiet Management möglichst frühzeitig weiterbilden. Ein chirurgischer
Chefarzt ohne Managerqualitäten und ohne eine ausgeprägte Fähigkeit zur Mitarbeiterführung und Kooperationsbereitschaft wird in Zukunft undenkbar sein.

Wir freuen uns, Sie zur Diskussion dieser und vieler weiterer hochaktueller Themen vom 24.04. bis 27.04.2012 in Berlin begrüßen zur dürfen.

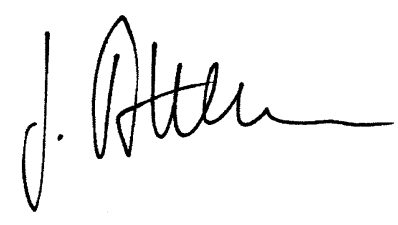

Dr. J. Fritzmann

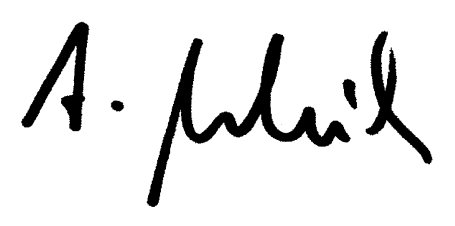

Prof. Dr. A. Ulrich

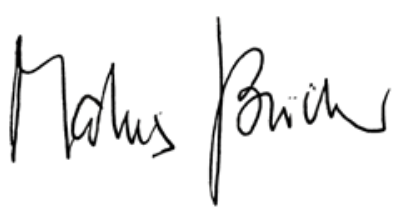

Prof. Dr. M.W. Büchler

\section{Korrespondenzadresse}

\section{Prof. Dr. M.W. Büchler}

Chirurgische Universitätsklinik, Klinikum der Universität Heidelberg, Im Neuenheimer Feld 110, 69120 Heidelberg Markus.Buechler@med.uni-heidelberg.de 